\title{
Méthodes de caractérisation des fibres de carbone en vue d'obtenir de bonnes conductivités électriques après insertion
}

\author{
G.-R. Bagga, S. Blazewicz (*) et $\mathrm{Ph}$. Touzain \\ Laboratoire d'Adsorption et Réaction de Gaz sur Solides, UA CNRS 413, E.N.S. Electrochimie et Electrométallur- \\ gie de Grenoble, I.N.P.G. B.P. 75, 38402 St Martin d'Hères, France
}

(Reçu le 21 juillet 1986, accepté le 7 octobre 1986)

\begin{abstract}
Résumé. - Des fibres de carbone provenant de différents précurseurs (PAN, BRAI-mesophase, méthane), traitées ou non traitées à haute température (HTT) ont été caractérisées par diverses méthodes physiques (diffraction des rayons $\mathrm{X}$, mesure des propriétés mécaniques et électriques) et électrochimiques dans le but de déterminer quelles seront les fibres dont les conductivités électriques obtenues après insertion de substances chimiques seront proches de celles des métaux les plus conducteurs. Les meilleures candidates sont les fibres vapodéposées (HTT $2900{ }^{\circ} \mathrm{C}$ ) et certaines fibres ex-BRAI-mésophase (P 55, HTT $\left.3300^{\circ} \mathrm{C}\right)$. Les fibres ex-PAN ne présentent pas les qualités requises pour donner des composés d'insertion ayant une bonne conductivité électrique.
\end{abstract}

\begin{abstract}
Several carbon fibres issued from different precursors (PAN, PITCH, methane) treated or not treated at high temperature (HTT) were characterized by different physical (XR diffraction, mechanical and electrical properties measurements) and electrochemical methods in order to ascertain the fibres for which intercalation of chemical compounds could give electrical conductivities as high as those metallic conductors. The better ones are the vapour grown fibres (HTT $2900^{\circ} \mathrm{C}$ ) and some ex-PITCH fibres (P 55, HTT $3300^{\circ} \mathrm{C}$ ). Ex-PAN fibres do not possess the qualities required to give for intercalation compounds with a good electrical conductivity.
\end{abstract}

\section{Introduction.}

Les fibres de carbone ou de graphite sont maintenant bien connues pour leurs propriétés mécaniques spécifiques de résistance à la traction et du module d'élasticité. Elles sont très compétitives et largement supérieures à celles des métaux pour certaines applications [1]. On sait d'autre part que l'insertion dans le graphite augmente en général la conductivité électrique qui approche dans certains cas celle de l'aluminium [2]. L'insertion dans les fibres de carbone afin d'améliorer leurs conductivités électriques jusqu'à une valeur intéressante tout en conservant leurs qualités mécaniques est un objectif que se sont donné plusieurs laboratoires. Cependant, avant l'insertion proprement dite, il serait souhaitable de connaître parfaitement les types de fibres en les caractérisant par diverses techniques physiques afin de pouvoir choisir les fibres qui pourront prétendre aux meilleurs performances à la fois mécaniques et électriques après insertion. On sait déjà [3] que

(*) Academy of Mining and Metallurgy, Institute of Materials Science 30-059 Krakow, Poland. les meilleures candidates sont celles dont la structure se rapproche le plus de celle du graphite pur ce qui incite à partir de fibres déjà assez bonnes conductrices de l'électricité et dans lesquelles l'insertion pourra se faire plus aisément.

Dans cette étude la caractérisation des fibres a été effectuée par diffraction des rayons $\mathrm{X}$, mesure de propriétés mécaniques et électriques et également par insertion électrochimique de l'acide sulfurique.

\section{Partie expérimentale.}

2.1 MATÉRIAUX DE DÉPART : LES FIBRES DE CARBONE. - Des échantillons des trois principales familles de fibres de carbone ont été utilisés pour ces caractérisations :

- les fibres à précurseurs textiles à base de polyacryonitrile (PAN),

- les fibres ex-mésophase de BRAI,

- les fibres vapodéposées (FVD) obtenues par décomposition catalytique de méthane à environ $1100^{\circ} \mathrm{C}[4]$. 
Tableau I. - Désignation des fibres et de leurs traitements thermiques.

[Designation of fibres with their heat treatment temperatures.]

\begin{tabular}{|c|c|c|c|c|c|c|c|c|}
\hline \multirow{3}{*}{ Précurseurs } & \multirow{3}{*}{$\begin{array}{c}\text { Producteurs } \\
\text { de la fibre }\end{array}$} & \multirow{3}{*}{$\begin{array}{l}\text { Désignation } \\
\text { commerciale }\end{array}$} & \multicolumn{6}{|c|}{ Fibres traitées } \\
\hline & & & \multicolumn{5}{|c|}{ HTT (sous argon) $\left({ }^{\circ} \mathrm{C}\right)$} & \multirow{2}{*}{$\begin{array}{c}\text { HTT } \\
2900^{\circ} \mathrm{C} \\
\text { sous poudre } \\
\text { de graphite }\end{array}$} \\
\hline & & & 2800 & 2900 & 3150 & 3300 & 3400 & \\
\hline PAN & $\begin{array}{l}\text { Celanese } \\
- \\
\text { Serofim } \\
- \\
\text { Hercules } \\
\text { Toray ind. } \\
- \\
\text { Cracovie }\end{array}$ & $\begin{array}{l}\text { Celion GY70 } \\
- \text { GY80 } \\
\text { Rigilor AX } \\
- \text { AG } \\
\text { Magnamite AS-4 } \\
\text { Torayca M40 } \\
-\quad \text { T300 } \\
\text { AGH }\end{array}$ & & GY70a & $\begin{array}{l}\text { AXa } \\
\text { AS-4a }\end{array}$ & & GY70b & $\begin{array}{l}\text { GY70a }\left({ }^{*}\right) \\
\text { GY80a }\left({ }^{*}\right) \\
\text { T300a }\left(^{*}\right) \\
\text { AGHa }\left(^{*}\right)\end{array}$ \\
\hline $\begin{array}{l}\text { BRAI- } \\
\text { MESO- } \\
\text { PHASE }\end{array}$ & $\begin{array}{l}\text { Union- } \\
\text { Carbide }\end{array}$ & \begin{tabular}{|c} 
Thornel P55 $\left(^{+}\right)$ \\
P75 \\
P75S \\
P100 \\
P120 \\
P100.4
\end{tabular} & & & P55a & P55b & & \\
\hline METHANE & $\begin{array}{l}\text { LARGS } \\
\text { (Grenoble) }\end{array}$ & FVD & FVDa & & & & & FVDa $(*)$ \\
\hline
\end{tabular}

$\left(^{+}\right)$Deux lots P55 ayant subi des traitements thermiques par le producteur ont été utilisés et désignés P55 (1) et P55 (2) (HTT de P55 (2) > HTT de P55 (1).

Le tableau I rassemble la liste des fibres employées dans ce travail et leur désignation.

Les fibres ex-PAN et ex-BRAI-mésophase ont été préparées industriellement. Un type de fibre ex-PAN provient du laboratoire académique des Mines et de Métallurgie de Cracovie.

Les fibres vapodéposées (FVD) ont été produites dans les conditions suivantes :

Gaz : méthane $25 \%$, hydrogène $75 \%$.

Débit : $250 \mathrm{ml} / \mathrm{min}$.

Diamètre et longueur du four : $35 \mathrm{~mm}$ et $100 \mathrm{~cm}$.

Température : $975^{\circ} \mathrm{C}$ au départ $(15 \mathrm{~min})$ suivi d'un chauffage à la vitesse de $1^{\circ} \mathrm{C}$ minn $^{-1}$ jusqu'à $1150^{\circ} \mathrm{C}$ maintenu durant $30 \mathrm{~min}$.

Catalyseur: nitrate ferrique déposé sur support en graphite exfolié (PAPYEX) à la concentration de $10^{-8} \mathrm{~g} \mathrm{Fe} / \mathrm{cm}^{2}$.

Le rendement en carbone déposé sous forme de fibres est de l'ordre de $10 \%$. Certaines des fibres obtenues ont atteint $20 \mathrm{~cm}$ de longueur et quelquefois $30 \mu \mathrm{m}$ de diamètre. La distribution des diamètres de ce lot de fibres est donnée en annexe.

Bien que la plupart des fibres commercialisées aient déjà subi un traitement thermique lors de leur élaboration, un certain nombre d'entre elles (Tabl. I) ont de nouveau été traitées à haute température sous circulation d'argon (HTT : heat treatment temperature).

Afin de les protéger de l'oxydation ou de la volatilisation pendant le traitement thermique, certaines de ces fibres ont été recouvertes durant le traitement à $2900^{\circ} \mathrm{C}$ par de la poudre de graphite (Ceylan, granulométrie $2 \mu \mathrm{m}$ ). Elles seront désignées par une $\left.{ }^{*}\right)$ comme indiqué sur le tableau I.

Toutes les fibres utilisées dans le cadre de cette étude ont été sélectionnées afin que leurs diamètres soient compris entre 8 et $10 \mu \mathrm{m}$.

L'aire exacte $S$ des sections des fibres a été déterminée de la façon suivante:

- fibres ex-PAN : leurs sections n'étant pas circulaires mais plutôt en forme de "haricots ", la mesure directe du diamètre à l'aide d'un microscope optique ne peut donner de résultats convenables directement. Afin de résoudre ce problème, les sections de fibres ont été photographiées au microscope électronique à balayage et les aires déterminées avec précision. Les valeurs obtenues sur plusieurs lots ont permis de trouver une relation linéaire entre le diamètre mesuré $d_{\mathrm{m}}$ par 
microscopie optique et la valeur correspondante du diamètre équivalent $d_{\mathrm{e}}$ à une section circulaire

$$
d_{\mathrm{e}}=1,27 d_{\mathrm{m}}-4,9(\mu \mathrm{m})
$$

- fibres ex-BRAI-mésophase : une détermination au microscope optique est suffisante car la section est circulaire et le diamètre ne varie pas sur la longueur.

- fibres FVD : leurs sections sont en général bien circulaires, par contre leurs diamètres peuvent varier le long de la longueur. Le diamètre moyen $d$ est obtenu par la relation :

$$
d=\frac{d_{1}+d_{2}+\cdots+d_{n}}{n}
$$

après $n$ mesures du diamètre $d$ le long de la fibre.

\subsection{APPAREILlaGE.}

2.2.1 Diffraction $X$. - La position et la largeur de la raie 002 permettent de calculer :

- le taux de graphitation $g$ par la relation de Maire et Mering [5] :

$$
g=\frac{3,44-d_{002}}{3,44-3,354}
$$

- la longueur de cohérence $L_{c}$ selon l'axe $c$ par la formule de Scherrer :

$$
L_{c}=\frac{0,89 \lambda}{\left[B^{2}-b^{2}\right]^{1 / 2} \cos \theta_{002}}
$$

dans laquelle :

$$
\begin{aligned}
\theta_{002}= & \text { angle de diffraction de la raie } 002, \\
d_{002}= & \text { distance réticulaire de la raie } 002: \\
& \lambda / 2 d \sin \theta \\
\lambda= & \text { longueur d'onde utilisée : } \lambda \mathrm{CuK}_{\alpha}= \\
& 1,562 \AA, \\
\mathrm{B}= & \text { largeur à mi-hauteur de la raie } 002(\text { en } 2 \theta), \\
b \quad= & \text { largeur instrumentale }\left(=0,15^{\circ}\right) .
\end{aligned}
$$

Les raies $h k o$ (raie 100 en particulier) sont de très faible intensité, très larges et souvent inexistantes et de ce fait nous n'avons pas pu obtenir de façon systématique la longueur de cohérence $L_{a}$.

2.2.2 Résistance à la traction et module d'Young.- Ils ont été déterminés à l'aide d'un appareil de traction (Instron 1122) dans les conditions suivantes :

- vitesse de la traverse : $2 \mathrm{~mm} / \mathrm{min}$,

- longueur initiale de la fibre monofilamentaire : $l$ $=25 \mathrm{~mm}$.

La force de rupture $F$ et l'allongement du fil au moment de la rupture $\Delta l$ permettent de calculer la résistance à la rupture $\sigma=F / S$ et le module d'élasticité $E=\sigma / \varepsilon$ avec $\varepsilon=\Delta l / l$ et $S=\pi d^{2} / 4$.

$\mathrm{La}$ résistance à la traction dépend fortement $\mathrm{du}$ diamètre de la fibre dans le cas des fibres vapodéposées. L'application de la statistique de Weibull compte tenu d'un certain nombre de simplifications [6] conduit à une relation simple entre $\sigma$ et $d$ :

$\ln \sigma=A-B \ln d \quad$ (avec $A$ et $B$ des constantes).

Le calcul de la résistance à la traction moyenne d'un lot de fibres FVD est exposé dans l'annexe.

2.2.3 Résistivité électrique. - La résistivité électrique est déterminée par la méthode à 4 points [7]. Le monofilament de carbone est fixé au niveau des quatre contacts par de la laque à l'argent. Les résultats ont été estimés sur une moyenne d'environ 10 mesures expérimentales (soit à $\pm 0,20 \mu \Omega \mathrm{m}$ ).

2.2.4 Insertion électrochimique de l'acide sulfurique. Une cellule à trois électrodes (travail en fibres de carbone, contre-électrode en platine et référence au sulfate mercureux ramenée sur les figures au potentiel de l'électrode au calomel saturée) plongées dans une solution d'acide sulfurique à $96 \%$ a été employée pour les essais d'insertion électrochimique par voltamétrie triangulaire ou cyclovoltamétrie.

La réaction électrochimique qui se produit au cours de l'insertion est la suivante :

$$
24 n \mathrm{C}+(p+1) \mathrm{H}_{2} \mathrm{SO}_{4} \leftrightharpoons \mathrm{C}_{24 n}^{+} \mathrm{HSO}_{4}^{-}\left(\mathrm{H}_{2} \mathrm{SO}_{4}\right)_{p}+\mathrm{H}^{+}+\mathrm{e}^{-}
$$

dans laquelle $n$ représente le stade du composé d'insertion et $p$ un coefficient stœchiométrique compris entre 2 et 3 .

\section{Résultats expérimentaux.}

3.1 Diffraction X. - Les spectres de diffraction ont été obtenus avec la raie $\mathrm{CuK} \alpha$ sur des mèches de plusieurs centaines de fibres. Les raies 002 de quelques fibres représentatives des 3 classes (ex-PAN, ex-BRAImésophase et FVD) sont représentées sur la figure 1.
On distingue nettement l'influence du traitement thermique sur la forme (intensité et largeur) et position de la raie. Le tableau II donne les valeurs du taux de graphitation $g$ et de la longueur de cohérence $L_{c}$ (ou épaisseur des cristallites selon l'axe $c$ ) calculées à partir des valeurs expérimentales $d_{002}$ et $B$. Le taux de graphitation $g$ est très sensible à HTT (voir par exemple la série GY 70 ou P 55). $L_{c}$ augmente aussi avec HTT mais des HTT supérieurs à $2800^{\circ} \mathrm{C}$ n'améliorent plus guère la valeur de $L_{c}$ comme cela a déjà été montré par ailleurs [8]. Le traitement des fibres sous lit 


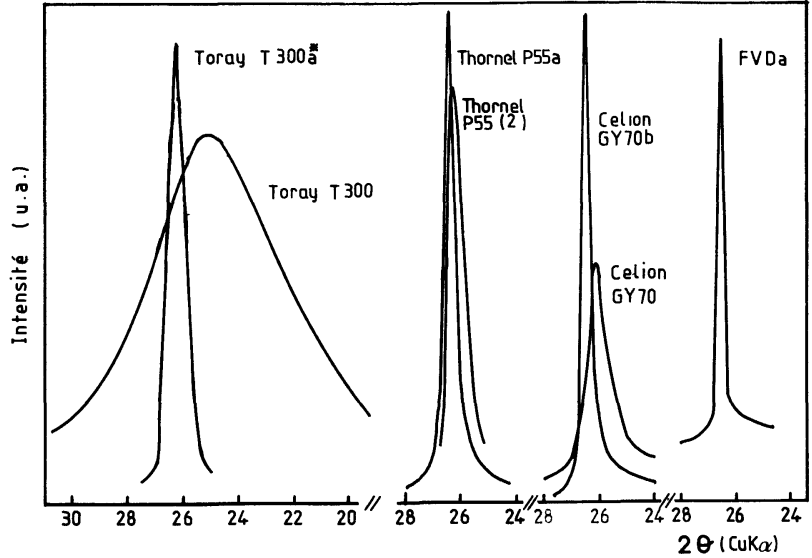

Fig. 1. - Profils des raies (002) obtenus par diffraction des rayons $\mathrm{X}(\mathrm{CuK} \alpha)$ sur différentes fibres de carbone avant et après traitement thermique.

[X Ray diffraction patterns (002 lines) of different carbon fibres before and after heat treatment $(\lambda \mathrm{CuK} \alpha)$.]

de poudre de graphite permettrait d'atteindre le même taux de graphitation que le traitement ordinaire à plus haute température : comparer les valeurs de $g$ pour les fibres GY 70a, GY 70a (*) et GY 70b respectivement de 56,93 et $90 \%$.

\subsection{InSERTION ÉlectrochimiQue De $\mathrm{H}_{2} \mathrm{SO}_{4} \cdot-\mathrm{Ce}$} test proposé initialement par Besenhard et al. [9] permet avec une technique relativement simple d'obtenir très rapidement de nombreux renseignements sur la nature et la disposition à l'insertion d'une fibre $[9,12]$.

Quelques cyclovoltamogrammes (CV) obtenus à la vitesse de $1 \mathrm{~V} \mathrm{~min}^{-1}$ sont représentés sur les figures 2 et 3. Ces voltamogrammes peuvent être caractérisés par plusieurs paramètres :

- Pics d'oxydation: leur nombre, leur amplitude (mA par mg de carbone) et leur largeur sont spécifiques du degré de cristallinité : dans le meilleur des cas, on distingue l'oxydation de la fibre successivement avec formation du $3^{\mathrm{e}}$ stade, $2^{\mathrm{e}}$ stade, $1^{\text {er }}$ stade puis sous forme d'oxyde graphitique (OG) enfin l'oxydation de l'eau avec départ d'oxygène. Noter l'influence du HTT sur le nombre et la forme des pics dans le cas par exemple de la fibre GY 70 (Fig. 2).

- Valeurs des potentiels d'oxydation: plus l'insertion est facile, plus les tensions d'oxydation sont faibles, observation souvent constatée. On peut définir ainsi le potentiel initial $(P I)$ correspondant au tout début de l'insertion, ce qui permet de quantifier par une valeur numérique l'aptitude à l'insertion de la fibre [10]. Cette valeur $P I$ varie de façon inversement proportionnelle au taux de graphitation. Dans le cas du $2^{\mathrm{e}} C V$ (Fig. 3) la valeur de $P I$ est légèrement inférieure à celle du $1^{\text {er }} C V$ par suite de la disparition d'un certain nombre de défauts au cours de la $1^{\text {er }}$ oxydation. La représenta-

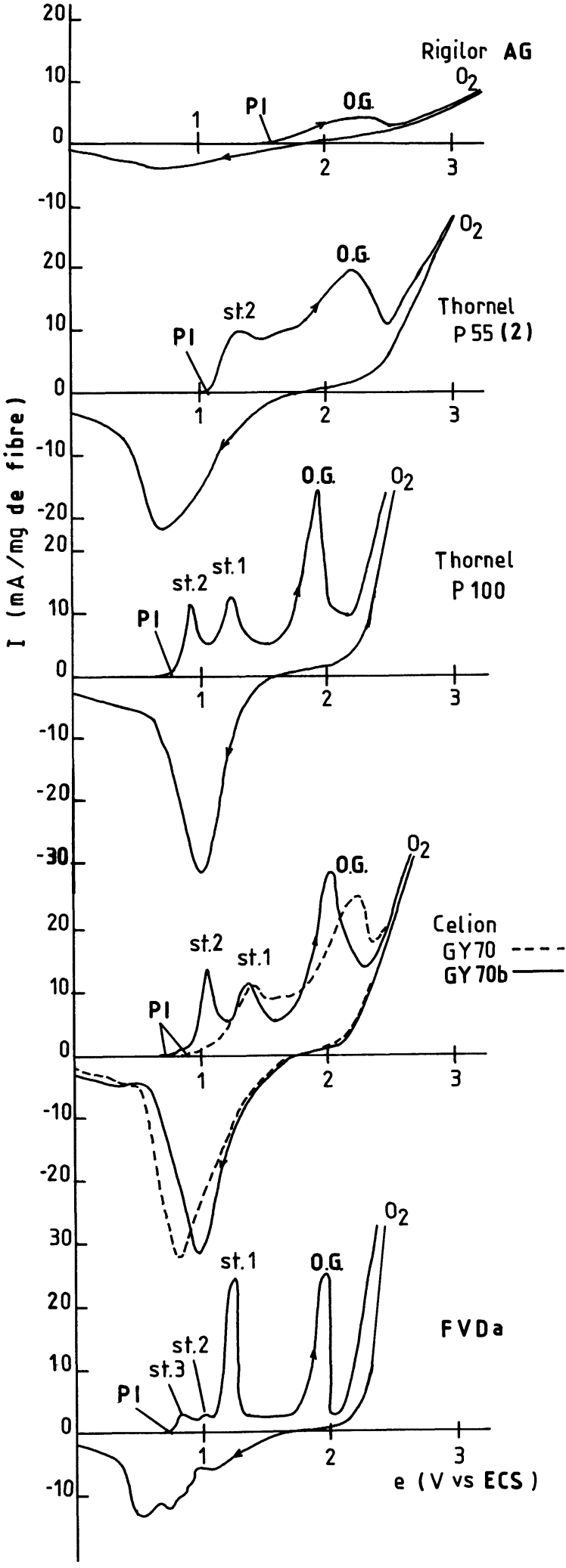

Fig. 2. - Cyclovoltamogrammes $\left(1^{\mathrm{er}}\right.$ cycle) de différentes fibres dans $\mathrm{H}_{2} \mathrm{SO}_{4}$ à $96 \%\left(v=1 \mathrm{~V} \mathrm{~min}^{-1}, P I=\right.$ Potentiel initial d'insertion par rapport au potentiel de l'électrode au calomel saturée).

[First cyclovoltamogram of different fibres in $96 \% \mathrm{H}_{2} \mathrm{SO}_{4}$ electrolyte $\left(v=1 \mathrm{~V} \mathrm{~min}{ }^{-1}, P I=\right.$ Initial Potential of Intercalation vs. SCE).] 
Tableau II. - Propriétés physico-chimiques des fibres utilisées dans cette étude.

[Physical and chemical properties of the fibres used in this study.]

\begin{tabular}{|c|c|c|c|c|c|c|c|c|}
\hline \multirow{2}{*}{ Fibres } & \multirow{2}{*}{$\begin{array}{l}\text { HTT } \\
\left({ }^{\circ} \mathrm{C}\right)\end{array}$} & \multirow{2}{*}{$\begin{array}{c}g \\
(\%)\end{array}$} & \multirow{2}{*}{$\begin{array}{r}L_{\mathrm{c}} \\
(\AA)\end{array}$} & \multirow{2}{*}{$\begin{array}{c}\rho_{\mathrm{a}} \\
(\mu \Omega \mathrm{m})\end{array}$} & \multicolumn{2}{|c|}{$P I(\mathrm{~V})$} & \multirow{2}{*}{$\begin{array}{c}\sigma \\
(\mathrm{MPa})\end{array}$} & \multirow{2}{*}{$\begin{array}{c}E \\
(\mathrm{GPa})\end{array}$} \\
\hline & & & & & er & $2^{e}$ & & \\
\hline GY70 & & 58 & 105 & 6,50 & 0,9 & 0,8 & 1860 & 517 \\
\hline GY70a & 2900 & 56 & 200 & 4,50 & - & - & - & - \\
\hline GY70a $(*)$ & 2900 & 93 & 200 & 3,60 & - & - & - & - \\
\hline GY70b & 3400 & 90 & 210 & 3,50 & 0,7 & 0,6 & 500 & 130 \\
\hline GY80 & & 34 & 95 & 6,40 & - & - & 1920 & 553 \\
\hline GY80a $\left(^{*}\right)$ & 2900 & 52 & 200 & 3,10 & - & - & - & - \\
\hline AX & & 0 & 18 & 15,50 & - & - & 2800 & 200 \\
\hline $\mathrm{AXa}$ & 3150 & 45 & 96 & 7,00 & - & - & - & - \\
\hline & & 22 & 45 & 11,00 & 1,6 & 0,85 & 2200 & 360 \\
\hline AS-4 & & 0 & 15 & 16,00 & - & - & 3590 & 234 \\
\hline AS-4a & 3150 & 45 & 95 & 6,30 & - & - & - & - \\
\hline M40 & & 5 & 50 & 8,00 & - & - & 2250 & 390 \\
\hline T300 & & 0 & 15 & 16,00 & - & - & 3140 & 230 \\
\hline T300a $\left(^{*}\right)$ & 2900 & 47 & 130 & 6,10 & - & - & - & - \\
\hline $\mathrm{AGH}$ & 1000 & 0 & 12 & 24,30 & - & - & - & - \\
\hline $\operatorname{AGHa}(*)$ & 2900 & 47 & 90 & 6,20 & - & - & - & - \\
\hline (1) & & 10 & 115 & 7,50 & 1,5 & 0,90 & 1800 & 390 \\
\hline P55 (2) & & 45 & 150 & 7,50 & 1,1 & 0,87 & 2150 & 380 \\
\hline P55a & 3150 & 75 & 170 & 2,50 & - & - & 1900 & 500 \\
\hline P55b & 3300 & 98 & 210 & 2,00 & 0,8 & 0,65 & 1900 & 580 \\
\hline P75 & & 47 & 140 & 5,00 & - & - & 2000 & 500 \\
\hline P75S & & 13 & 150 & 5,00 & - & - & 2100 & 520 \\
\hline P100 & & 91 & 140 & 2,60 & 0,8 & 0,65 & 2200 & 700 \\
\hline P120 & & 51 & 190 & 2,20 & - & - & 2200 & 820 \\
\hline P100-4 & & 66 & 340 & $(1,20)$ & - & - & - & - \\
\hline FVD & 1100 & 0 & 25 & 11,00 & - & - & 2000 & 250 \\
\hline FVDa & 2800 & 99 & 500 & $0,70 \pm 0,20$ & 0,75 & 0,65 & 3500 & 270 \\
\hline FVDa $\left(^{*}\right)$ & 2900 & 93 & 430 & $0,50 \pm 0,20$ & - & - & - & - \\
\hline HOPG & 3000 & 100 & $\sim 1000$ & 0,40 & 0,65 & 0,65 & - & - \\
\hline
\end{tabular}

HTT = Heat Treatment Temperature (température de traitement pour la graphitation).

$g \quad=$ taux de graphitation.

$L_{c} \quad=$ longueur de cohérence selon l'axe $c$ (il permet de calculer le nombre $n$ de plans de graphite contigus par $\left.n \simeq L_{c} / 3,4\right)$.

$\rho_{\mathrm{a}} \quad=$ résistivité électrique de la fibre.

$P I \quad=$ potentiel initial d'insertion pour la $1^{\text {re }}$ ou la $2^{\mathrm{e}}$ cyclovoltammétrie $(V$ vs. ECS).

$\sigma \quad=$ résistance à la traction.

$E \quad=$ module d'élasticité.

HOPG $=$ Highly Oriented Pyrographite (Pyrographite de grande qualité cristalline).

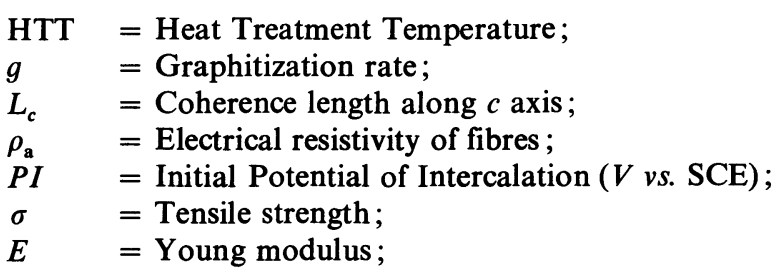

HOPG = Highly Oriented Pyrographite. 


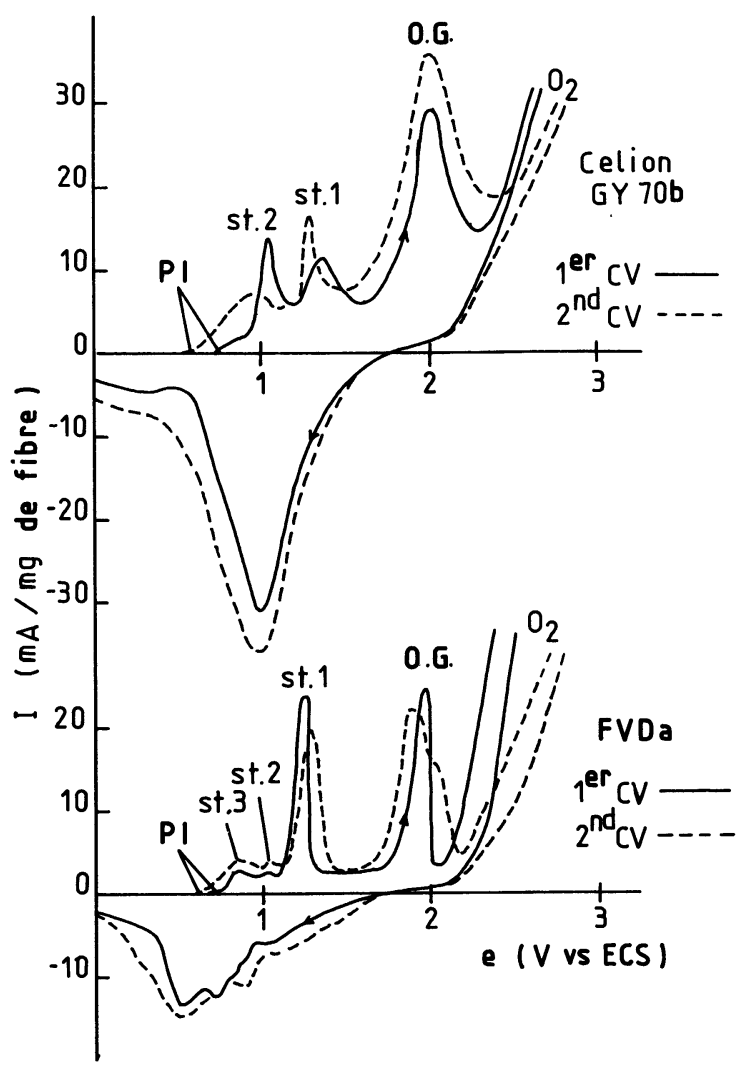

Fig. 3. - Premier et second cycles de voltamétrie dans $\mathrm{H}_{2} \mathrm{SO}_{4}$ à $96 \%$ de deux types de fibres traitées à haute température.

[First and second cyclovoltamograms in $96 \% \mathrm{H}_{2} \mathrm{SO}_{4}$ electrolyte of two kinds of fibres treated at high temperature.]

tion du $P I$ du $2^{\mathrm{e}} C V$ est une fonction quasi linéaire du taux de graphitation $g$ (Fig. 4).

- Pics de réduction : un seul pic de réduction est en général obtenu au cours du retour de la $C V$. Il

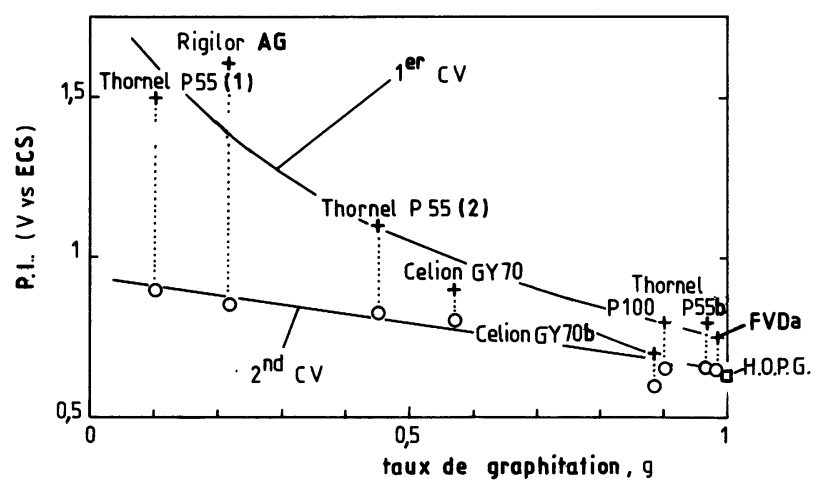

Fig. 4. - Potentiel initial d'insertion $(P I)$ du premier et du second cyclovoltamogrammes de différentes fibres en fonction de leur taux de graphitation.

[First and second cyclovoltamograms PI of different fibres as a function of the graphitization rate.] correspond à la réduction de l'OG confondue avec le retour à un stade supérieur ou désinsertion. Dans le cas des fibres bien graphitées (FVDa) on observe plusieurs pics successifs quoique assez mal séparés. L'arrêt de l'oxydation à un potentiel inférieur à celui de la formation de l'OG, permet d'observer des pics de réduction correspondant à la désinsertion seule [11].

3.3 PROPRIÉtÉS MÉCANIQUES. - Quelques valeurs de résistance à la rupture et du module d'Young sont données sur le tableau II. Cependant, aucune corrélation nette ne permet de relier ces valeurs aux taux de graphitation ou au PI ni non plus au HTT. Des valeurs élevées sont évidemment souhaitées puisque l'on sait que au cours de l'insertion, ces valeurs auront plutôt tendance à diminuer. Il semble en tout cas, comme nous l'avons observé par exemple pour les fibres GY $70 \mathrm{~b}$ et $\mathrm{P} 100.4$, qu'un HTT supérieur à $3000-3200^{\circ} \mathrm{C}$ à pour effet de fragiliser les fibres qui se cassent alors beaucoup plus facilement par flexion d'où l'intérêt du traitement thermique à la plus basse température possible et sous lit de poudre protectrice.

3.4 ConduCTIVITÉ ÉLECTRIQUE. - Les valeurs des résistivités électriques de chacune des fibres sont indiquées sur le tableau II. Comme cela a déjà été montré [8, 13-16], la conductivité croît avec HTT c'est-à-dire en fait avec le taux de graphitation. Sur la figure 5 qui donne les valeurs des résistivités en fonction de $g$, on pourra constater d'une part qu'effectivement les résistivités diminuent quand HTT augmente (pour les fibres indicées a ou b) et d'autre part que la résistivité est globalement inversement proportionnelle au taux de graphitation (ce taux n'étant qu'un coefficient purement empirique, il n'y a pas à rechercher de relation linéaire entre $\rho$ et $g$ ).

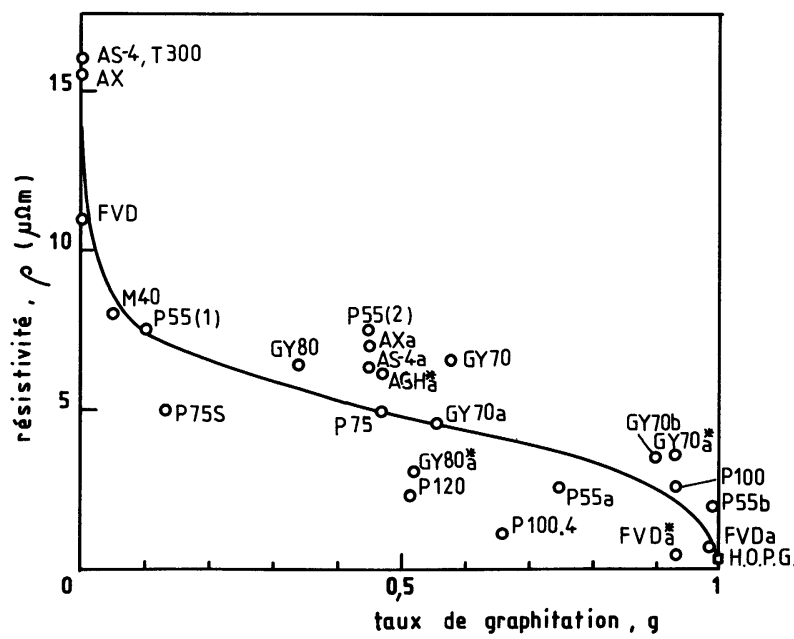

Fig. 5. - Valeurs des résistivités de différentes fibres en fonction de leur taux de graphitation.

[Resistivities of different fibres versus graphitization rate.] 
Il semble cependant plus justifié de relier la résistivité de la fibre $\rho$ au nombre de défauts rencontrés dans le plan $(a, b)$.

$\mathrm{La}$ relation empirique

$$
\rho=\rho_{\mathrm{u}}+\rho_{\mathrm{b}} \frac{a}{L_{a}}
$$

dans laquelle $\rho_{\mathrm{u}}$ est la résistivité des cristallites de la fibre, $\rho_{\mathrm{b}}$ est la résistivité des défauts d'épaisseur $a$ reliant les cristallites entre eux, a été proposée par Guigon et al. [14]. Cette relation est valable pour un certain nombre de fibres haut module et haute résistance du type PAN [14].

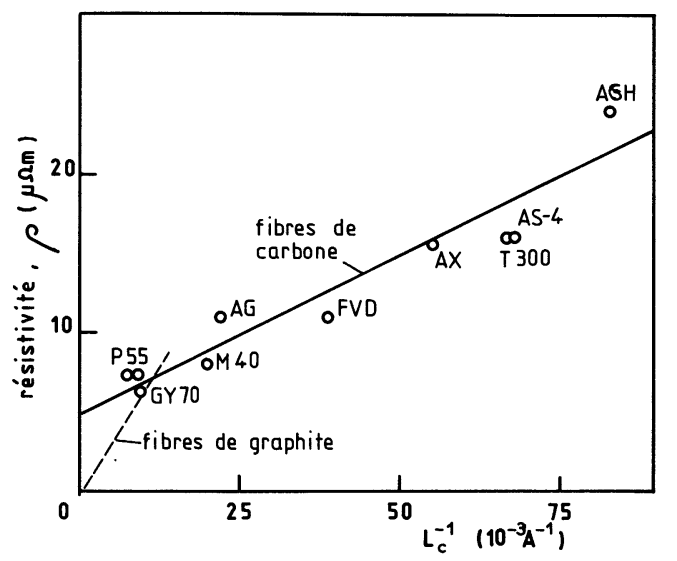

Fig. 6a. - Valeurs des résistivités de différentes fibres de carbone (non traitées à haute température) en fonction de $1 / L_{c}$.

[Resistivities of different carbon fibres (no heat treated) as a function of $1 / L_{c}\left(L_{c}\right.$ : Coherence length along $c$ axis).]

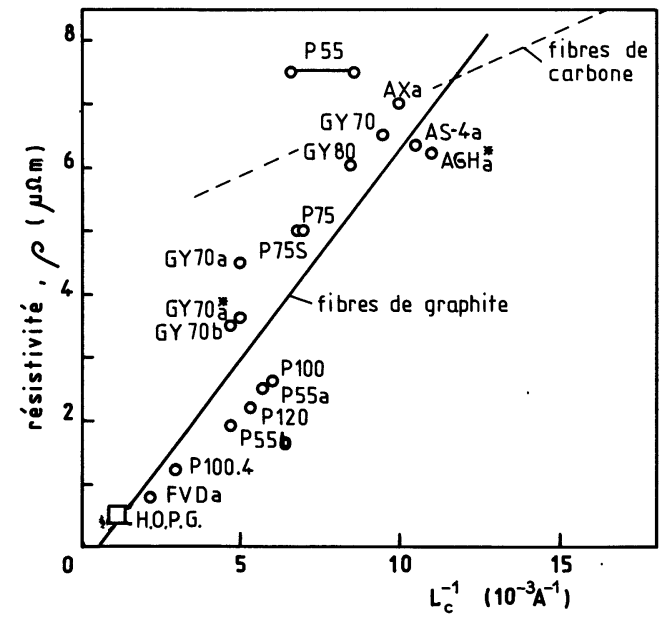

Fig. 6b. - Valeurs des résistivités de différentes fibres de graphite (traitées à haute température) en fonction de $1 / L_{c}$.

[Resistivities of different graphite fibres (heat treated) as a function of $1 / L_{c}$.]
En supposant de façon relativement arbitraire que le nombre de défauts rencontrés dans le plan de base ( $a$, b) est proportionnel aussi à $1 / L_{c}$ puisque $L_{a}$ et $L_{c}$ sont pratiquement proportionnels (voir par exemple la Fig. 4 de Réf. [13]), nous avons trouvé une relation linéaire entre $\rho$ et $1 / L_{c}\left(L_{c}\right.$ en $\left.\AA\right)$ dans le cas des fibres PAN, BRAI-mésophase et FVD non traitées (Fig. 6a) :

$$
\rho(\mu \Omega \mathrm{m})=4,86+203 / L_{c}(R=0,96)
$$

et dans le cas de ces mêmes fibres après traitement thermique (Fig. 6b) :

$$
\rho(\mu \Omega \mathrm{m})=-0,4+677 / L_{c}(R=0,94) .
$$

Dans le premier cas, la valeur $\rho_{\mathrm{u}}(\sim 5 \mu \Omega \mathrm{m})$ est très grande comparée à celle du graphite pur $(0,4 \mu \Omega m)$ prouvant que les fibres non traitées sont très mal graphitées. Par contre, dans le deuxième cas, la valeur $\rho_{\mathrm{u}}$ aux erreurs expérimentales ou statistiques près est proche de celle du graphite HOPG. Les fibres traitées à haute température sont donc bien graphitées. Seuls les défauts dus aux joints de grains sont les causes des valeurs de résistivité résiduelle.

On peut également constater sur le tableau II que les valeurs de la résistivité sont approximativement corrélées aux valeurs du potentiel initial d'insertion $(P I)$. On remarque là aussi que la méthode électrochimique donne des indications qui peuvent être équivalentes en information aux mesures de résistance électrique pour désigner les meilleures fibres.

\section{Discussion et conclusion.}

Trois types de fibres de carbone (fibres ex-PAN ; fibres ex-BRAI-mésophase et fibres vapodéposées exméthane) ont été caractérisés en vue de rechercher celles qui pourraient donner des conducteurs électriques intéressants après insertion chimique. Les méthodes de caractérisation utilisées ont été la diffraction des rayons $\mathrm{X}$, la cyclovoltamétrie au cours de l'insertion électrochimique de l'acide sulfurique, les mesures de résistivité électrique, de résistance à la traction et du module d'Young.

Tous les résultats expérimentaux indiquent que les fibres les mieux graphitées avec le moins de défauts possible dans les conditions les plus avantageuses pour préserver leurs propriétés mécaniques (c'est-à-dire aux alentours d'HTT de $2800-3000^{\circ} \mathrm{C}$ et de préférence sous poudre de graphite) et qui possèdent à la fois une très faible résistivité électrique sont les candidates les plus intéressantes pour l'insertion chimique en vue d'obtenir de bons conducteurs électriques comme d'ailleurs l'ont montré de récents travaux [3, 13, 14]. Les fibres vapodéposées (FVDa) semblent à ce point de vue les meilleures. La série de fibres ex-BRAImésophase de Thornel permettrait d'obtenir des résultats encore intéressants (à noter qu'à l'heure actuelle ces dernières sont les seules produites convenablement 
en grande longueur). Il apparaît cependant que certaines d'entre elles ont subi un HTT trop grand qui les fragilise beaucoup trop (en particulier à la flexion) et les rendent inaptes à l'utilisation envisagée. Les fibres ex-PAN ont une résistance électrique trop élevée et un taux de graphitation trop faible pour être employées. Seule la fibre Celion GY 70 traitée à très haute température pourrait être utilisée mais là aussi une température de traitement trop élevée fragilise la fibre dans des conditions qui ne seraient plus acceptables.

En résumé, une bonne fibre de carbone qui serait apte à l'insertion en conduisant à une très bonne conductivité électrique devrait atteindre pour ses propriétés physicochimiques les valeurs ci-dessous déduites empiriquement de nos résultats expérimentaux :

- taux de graphitation $g \geqslant 80 \%$ (ou $d_{002}<3,37 \AA$ ),

- longueur de cohérence $L_{c} \geqslant 150 \AA$,

- résistivité initiale $\rho_{\mathrm{a}}<3 \mu \Omega \mathrm{m}$,

- potentiel initial d'insertion dans l'acide sulfurique concentré et pour le $1^{\text {er }}$ cycle $P I<0,8 \mathrm{~V}$ vs. ECS,

- résistance à la traction, ) selon les applications

- module d'Young $\} \begin{aligned} & \text { demandées mais les plus } \\ & \text { grandes possibles. }\end{aligned}$

Les conclusions de cet article sont en accord avec les résultats expérimentaux de mesure de la résistivité après insertion (pour lequel on trouvera quelques références significatives dans les articles [ 3 et 13]. La meilleure résistivité atteinte est à l'heure actuelle de $0,01 \mu \Omega \mathrm{m}$ [17] pour une fibre type FVDa insérée avec $\mathrm{AsF}_{5}$.

\section{Remerciements.}

Les auteurs sont reconnaissants à Monsieur SerraTosio qui a mis à notre disposition l'appareil de traction de l'Ecole Française de Papeterie de Grenoble et à la Société Le Carbone Lorraine pour les traitements de graphitation des fibres.

Annexe.

RÉSISTANCE A LA TRACTION D'UN LOT DE FIBRES FVD NON TRAITÉES A HAUTE TEMPÉRATURE. - La détermination du diamètre moyen des fibres de ce lot a été effectuée par microscopie optique sans risque d'erreur importante. La distribution des diamètres compris entre $2 \mu \mathrm{m}$ et $28 \mu \mathrm{m}$ de ce lot comportant 225 fibres est présentée sur l'histogramme de la figure 7. Le diamètre moyen a pu ainsi être évalué à 5,9 $\mu \mathrm{m}$.

La résistance jusqu'à rupture de 40 fibres appartenant à des tranches de section différente de ce lot a été mesurée et les valeurs moyennes de ces résistances ont été tracées en fonction du diamètre correspondant en coordonnées logarithmiques sur la figure 8. Une relation linéaire (justifiable par la statistique de Weibull) entre la résistance à la traction (en $\mathrm{MPa}$ ) et le diamètre $d$ (en $\mu \mathrm{m})$ donne :

$$
\log \sigma=4,26-1,05 \log d \text {. }
$$

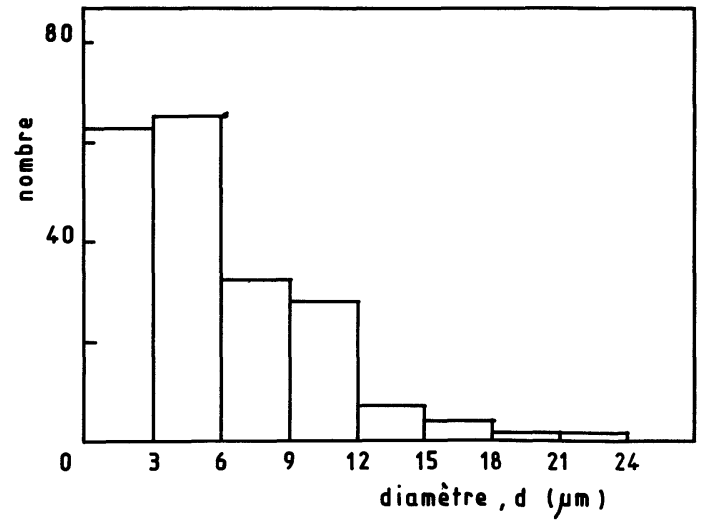

Fig. 7. - Histrogramme d'un lot de fibres vapodéposées (FVD) en fonction de leurs diamètres.

[Histogram of a lot of vapour grown fibres (FVD) versus diameter.]

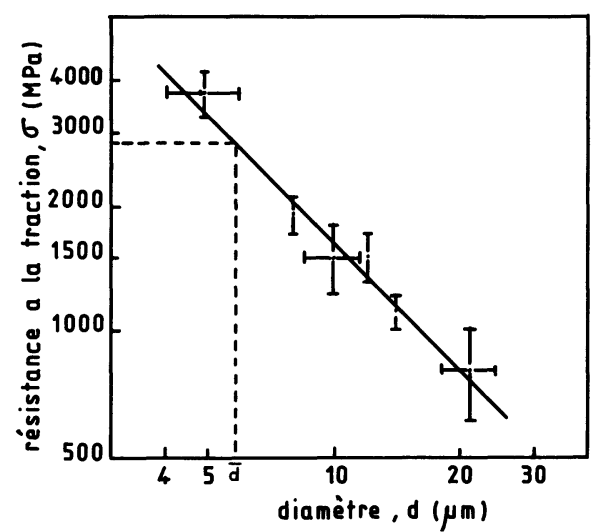

Fig. 8. - Résistance à la traction d'un lot de fibres vapodéposées (FVDa) en fonction de leurs diamètres.

[Tensile strength of a lot of vapour grown fibres (FVDa) versus diameter.]

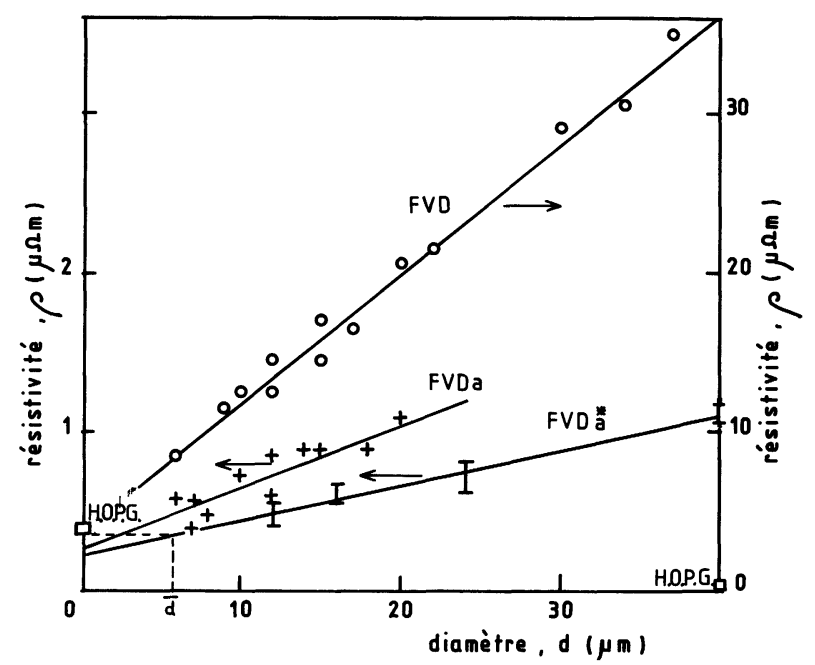

Fig. 9. - Résistivité électrique des fibres vapodéposées non traitées (FVD) ou traitées à haute température (FVDa et FVDa $\left({ }^{*}\right)$ ) en fonction de leurs diamètres.

[Electrical resistivities of vapour grown fibres no heat treated (FVD) or heat treated (FVDa and FVDa $\left(^{*}\right)$ ) versus diameter.] 
Le diamètre moyen étant connu, on peut donc estimer la valeur moyenne de la résistance à la traction de ce lot à $2800 \mathrm{MPa}$. Rappelons que les valeurs indiquées sur le tableau II correspondent seulement aux fibres de diamètre compris entre 8 et $10 \mu \mathrm{m}$.

RÉSISTANCE ÉLECTRIQUE D'UN LOT DE FIBRES FVD NON TRAITÉES ET TRAITÉES A HAUTE TEMPÉRATURE. - Les résistivités électriques des fibres du lot précédent ont été mesurées en fonction de leur diamètre. La figure 9 présente l'ensemble des résultats pour les FVD non traitées [18], les FVD traitées à $2800^{\circ} \mathrm{C}$ [19] et celles du lot utilisé dans cette étude, traitées à $2900{ }^{\circ} \mathrm{C}$ sous poudre de graphite. La variation de $\rho$ est pratiquement linéaire en fonction du diamètre $d$ (en $\mu \mathrm{m})$ et obéit aux lois suivantes : $\rho($ FVD $) \quad=0,82 d+3,61(\mu \Omega \mathrm{m})(R=0,99)$

$\rho$ (FVDa) $=0,042 d+0,233(R=0,90)$

$\rho($ FVDa $(*))=0,021 d+0,250(R+0,99)$.

L'ensemble du lot FVDa (*) de diamètre moyen $5,9 \mu \mathrm{m}$ dont les résistivités varient entre 0,3 et $1 \mu \Omega \mathrm{m}$ aurait donc une résistivité moyenne de l'ordre de $0,40 \mu \Omega \mathrm{m}$ équivalente à celle d'un bon pyrographite.

Remarquons que la variation de la résistivité en fonction du diamètre ne correspond pas aux résultats de Tahar et al. [20] qui trouvent une influence du diamètre en $1 / d$ pour $d<8 \mu \mathrm{m}$ dans le cas des fibres FVD à précurseur benzène. La température de traitement $\left(\mathrm{HTT}=3500^{\circ} \mathrm{C}\right.$ ) en graphitant fortement le dépôt pyrolytique de la fibre pourrait seule expliquer la cause de cette différence essentielle.

\section{Bibliographie}

[1] FISCHARD, D. B., Sciences and new applications of carbon fibers, Internat Symposium Toyohashi University (1984) p. 53.

[2] Spain, I. L., Chemistry and physics of carbon. Walker and Thrower, ed., (M. Dekker) 16 (1981) p. 119

[3] Touzain, Ph., Bagga, G. R. et Blazewicz, S., Synth. Metals (à paraître).

[4] Benissad, F., Gadelle, P., Coulon, M. et BonneTAIN, L., 4th International Carbon conference, Baden-Baden June 30-July 4, 1986, p. 709.

[5] Maire, J. et Mering, J., Les Carbones Pacault, ed. (Masson) 1965, p. 129.

[6] Chwastiak, S., BarR, J. B. et Didchenko, R., Carbon 17 (1979) 49.

[7] Coleman, L. B., Rev. Sci. Instrum. 46 (1975) 1125.

[8] Bright, A. A. et Singer, L. S., Carbon 17 (1979) 59.

[9] Besenhard, J. O., Mohwald, H. et Nickl, J. J., Rev. Chim. Min. 19 (1982) 588.

[10] Touzain, Ph. et BAgGA, G. R., Sciences and new applications of carbon fibers, Internat Symposium, Toyohashi University (1984) 91.
[11] Maeda, Y., OKemoto, Y. et InAGaki, M., J. Electrochem. Soc. 132 (1985) 2369.

[12] Olk, C. H. et Heremans, J. P., Synt. Metals 12 (1985) 511.

[13] Murday, J. S., Dominguez, D. D., Moran, J. A., LEe, W. D. et EAton, R., Synt. Metal 9 (1984) 397.

[14] Guigon, M., Oberlin, A. et Desarmot, G., Internat. Carbon Conference Bordeaux (1984) 170.

[15] Matsumura, K., Takahashi, A. et Tsukamoto, J., Synt. Metals 11 (1985) 9.

[16] Heremans, J. P., Carbon 23 (1985) 431.

[17] Shioya, J., Matsubara, H. et Murakami, S., Synt. Metals 14 (1986) 113.

[18] Kandani, N., Thèse INPG, Grenoble (1983).

[19] BaggA, G. R., Thèse INPG, Grenoble (1985).

[20] Tahar, M. Z., Dresselhaus, M. S. et Endo, M., Carbon 24 (1986) 67. 